\title{
Ingestión de cuerpo extraño (batería de botón) con presentación atípica en el lactante. Reporte de caso y revisión de la bibliografía
}

\author{
Foreign body ingestion (button battery) with atypical \\ presentation in infancy. A case report and literature review
}

\author{
Lina Margarita Baños-Rocha, ${ }^{1}$ Karen Rubí Ignorosa-Arellano, ${ }^{2}$ Ana Isabel Quesada-Tortoriello, ${ }^{3}$ José Francisco \\ Cadena-León, ${ }^{2}$ Erick Manuel Toro-Monjaraz, ${ }^{2}$ Flora E. Zárate-Mondragón, ${ }^{2}$ Alejandro Loredo-Mayer, ${ }^{2}$ Roberto \\ Cervantes-Bustamante, ${ }^{2}$ Jaime Alfonso Ramírez-Mayans ${ }^{2}$
}

\begin{abstract}
Resumen
ANTECEDENTES: El estridor es un sonido respiratorio, de tono alto, generado por el flujo turbulento de aire a través de una vía aérea estrecha, con diagnóstico diferencial que incluye procesos infecciosos y no infecciosos, incluida la ingestión de cuerpos extraños. CASO CLínICO: Lactante de 11 meses de edad, previamente sano, con estridor súbito y rechazo de la alimentación de 5 días de evolución. Recibió 2 esquemas antimicrobianos sin mejoría, de manera fortuita se detectó, en la radiografia de tórax, un objeto redondeado, radiopaco, localizado en el tercio superior del esófago. Por vía endoscópica se extrajo un cuerpo extraño, tipo pila de botón, que dejó una lesión ulcerada con áreas de necrosis (Zargar IIB). Se trató con antibióticos de amplio espectro, esteroides y nutrición parenteral. Su evolución fue satisfactoria, sin complicaciones. En la endoscopia de control a las 6 semanas se advirtió una cicatriz en la zona afectada.

CONCLUSIONES: El estridor agudo y la tos acompañados de manifestaciones gastrointestinales: vómito y rechazo a la alimentación en lactantes y preescolares sin antecedente de infección, obligan a descartar la existencia de cuerpos extraños y a practicar estudios de extensión y extracción endoscópica oportuna, sobre todo en el caso de las baterías de botón.
\end{abstract}

PALABRAS CLAVE: Estridor; sonido respiratorio; cuerpo extraño; aspiración; tratamiento con antibiótico; radiopaco; esófago; nutrición parenteral total

\section{Abstract}

BACKGROUND: Stridor is a high-pitched breath sound resulting from turbulent air flow in the narrowing areas of the large airways. A wide variety of differential diagnoses must be considered in the pediatric patient with stridor, including infectious and noninfectious causes, being foreign body aspiration as one of the etiologies to consider.

CLINICAL CASE: Previously healthy 11-month-old infant with sudden stridor and 5-dayold refusal of food. He received 2 antimicrobial schemes without improvement, in a fortuitous way it was detected, in the thorax radiography, a rounded object, opaque radio, located in the superior third of the esophagus. A foreign body, similar to a button battery, was removed endoscopically, leaving an ulcerated lesion with areas of necrosis (Zargar IIB). It was treated with broad-spectrum antibiotics, steroids and parenteral nutrition. His progress was satisfactory, without complications. In the control endoscopy at 6 weeks a scar was noticed in the affected area.

CONCLUSIONS: Cough and acute-onset stridor, followed by gastrointestinal manifestations such as vomiting and feeding refusal in infants and toddlers without a history of infectious disease, forced to rule out the presence of a foreign body, additional evaluation and endoscopic removal in an appropriate time.

KEYWORDS: Stridor; Breath sound; Foreign body; Aspiration; Antibiotic treatments; Radio opaque; Esophagus; Total parenteral nutrition

\begin{abstract}
${ }^{1}$ Residente de segundo año de la especialidad de Gastroenterología y Nutrición pediátrica.

${ }^{2}$ Servicio de Gastroenterología y Nutrición Pediátrica.

${ }^{3}$ Residente de primer año de la especialidad de Gastroenterología y Nutrición pediátrica.
\end{abstract}

Instituto Nacional de Pediatría, Ciudad de México.

Recibido: 19 de septiembre 2019

Aceptado: 27 de octubre 2020

Correspondencia

Karen Rubí Ignorosa-Arellano

karenignorosaa@gmail.com

Este artículo debe citarse como Baños-Rocha LM, Ignorosa-Arellano KR, Quesada-Tortoriello Al, Cadena-León JF, Toro-Monjaraz EM, Zárate-Mondragón $\mathrm{FE}$, Loredo-Mayer A, CervantesBustamante R, Ramírez-Mayans JA. Ingestión de cuerpo extraño (batería de botón) con presentación atípica en el lactante. Reporte de caso y revisión de la bibliografía. Acta Pediatr Méx 2020; 257-63. 


\section{ANTECEDENTES}

El estridor es un sonido respiratorio de tono alto, generado por un flujo rápido y turbulento de aire a través de estrechamientos de la vía aérea superior (regiones supra y subglótica, laringe y tráquea proximal). ${ }^{1,2,3}$ Es uno de los motivos de consulta más frecuentes en los servicios de urgencias pediátricas, en todas las edades, con diagnóstico diferencial que incluye causas infecciosas y no infecciosas; por esto, sin duda, la historia clínica y el examen físico son los pilares para orientar el diagnóstico y tratamiento. ${ }^{3}$

Las fases del estridor sirven para establecer el alcance de la obstrucción. Si es inspiratoria el origen es dinámico por encima de las cuerdas vocales. Si es espiratoria la localización puede ser intratorácica y si es bifásica sugiere una obstrucción fija de la vía aérea, a la altura de la glotis, subglotis o parte superior de la tráquea. ${ }^{1-4}$

El estridor agudo obliga a descartar, en primer lugar, un proceso infeccioso y, en casos sin datos de infección previa o actual, debe practicarse una broncoscopia para el estudio integral de las causas obstructivas de la vía aérea. También debe considerarse la ingestión de un cuerpo extraño en la vía respiratoria como diagnóstico diferencial. Se han descrito casos de cuerpo extraño localizados en la parte superior del esófago, con signos y síntomas compatibles con obstrucción de la vía aérea secundarios a una lesión adyacente del nervio laríngeo recurrente, como sucedió en el caso que se reporta. ${ }^{4,5,6}$

\section{CASO CLÍNICO}

Lactante de 11 meses, previamente sano, con estridor y tos de inicio súbitos de 5 días de evolución. En dos ocasiones se valoró en consulta externa, con indicación de tratamiento con antibióticos de amplio espectro y antitusígenos, sin mejoría. 24 horas antes de hospitalizarlo tuvo rechazo de la vía oral y vómito. En la radiografía de tórax se identificó una imagen redondeada, radiopaca, en el tercio superior del esófago; por ese motivo fue enviado al Instituto Nacional de Pediatría.

A la exploración física manifestó irritabilidad, sialorrea y estridor inspiratorio que se agravaban con el llanto, sin evidencia de datos de dificultad respiratoria, con oximetría de pulso de $98 \%$, sin evidencia de otras alteraciones. La radiografía de tórax anteroposterior y lateral confirmó la existencia de un cuerpo extraño de $25 \mathrm{~mm}$ de diámetro, a la altura del esófago cervical, radioopaco, de apariencia "escalonada" en la imagen lateral, compatible con el diagnóstico de ingestión de pila de botón (Figura 1). Se practicó una endoscopia de urgencia, con anestesia general, para extraerle el cuerpo extraño, tipo batería de botón, de $2.5 \mathrm{~cm}$ de diámetro, que se localizaba a $10 \mathrm{~cm}$ de la arcada dental superior (Figura 2). En ese mismo sitio se observó una lesión ulcerada, profunda, ubicada en la cara posterior del esófago de aproximadamente $2 \mathrm{~cm}$ de diámetro, cubierta con exudado fibrinoide, con algunas zonas de necrosis. Se utilizó la clasificación de esofagitis por caústicos de Zargar, por los mecanismos de lesión asociados con la batería de botón, se catalogó con grado IIB (Figura 3A), sin lesiones estomacales o duodenales.

Se le indicó tratamiento médico conforme al lineamiento institucional del servicio de Gastroenterología y Nutrición con 1 g/1.73 m²/día de metilprednisolona durante 3 días, $100 \mathrm{mg} /$ $\mathrm{kg} / \mathrm{d}$ de ceftriaxona durante 10 días y ayuno con nutrición parenteral total. Al tercer día de estancia hospitalaria fue valorado por el otorrinolaringólogo, debido al estridor; se le practicó una nasofibroendoscopia en la que se identificó paresia de la cuerda vocal derecha; se indicó 

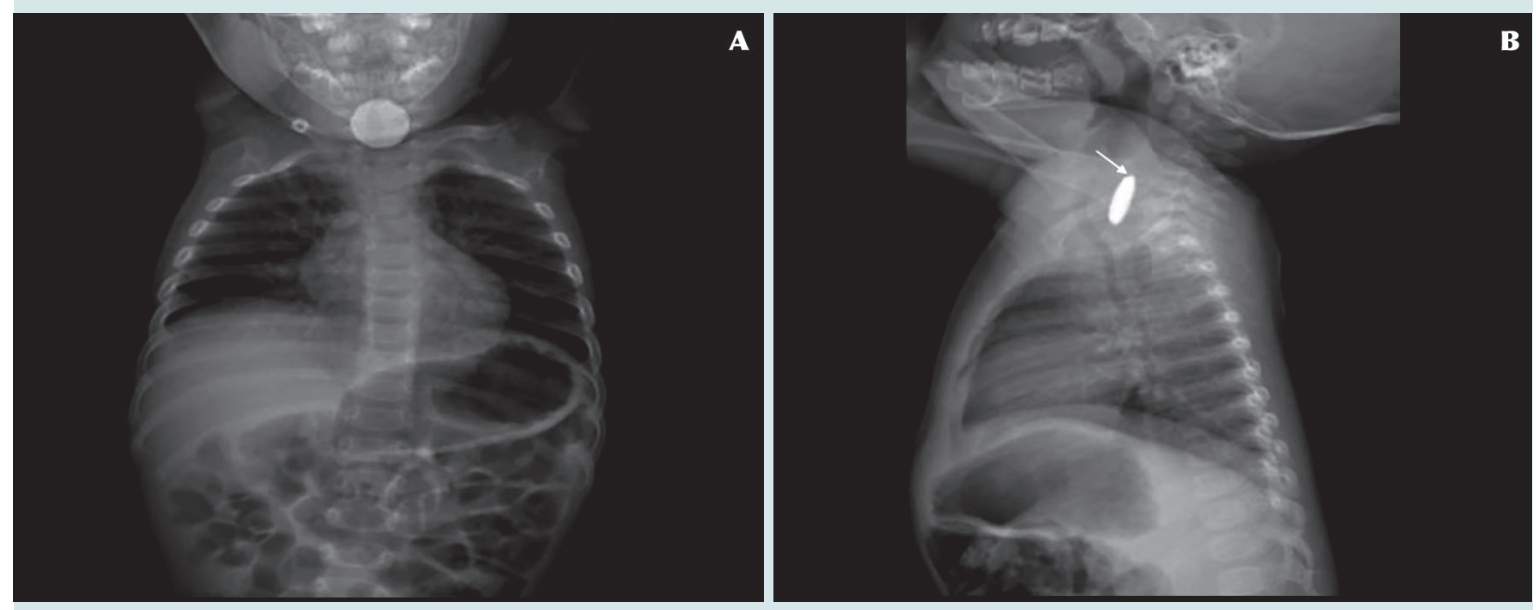

Figura 1. A) Radiografía de tórax AP con cuerpo extraño alojado en el esófago cervical. B) Radiografía de tórax lateral con objeto con imagen "escalonada" o step off que se señala con la flecha blanca.

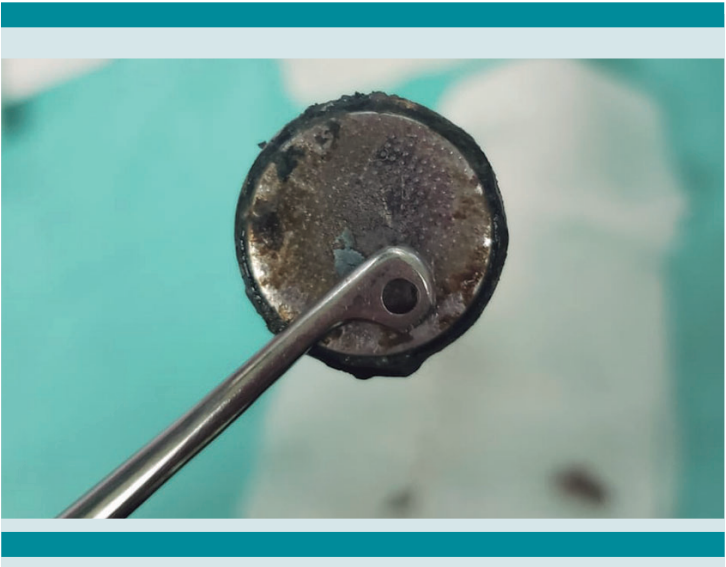

Figura 2. Cuerpo extraño (batería de botón) después de haberse removido del esófago, con corrosión notable en la superficie.

tratamiento conservador. Debido a esta evidencia se omitió la broncoscopia, como evaluación integral de la vía aérea.

A las dos semanas se le practicó una endoscopia, en la que se advirtió una úlcera cicatrizada con tejido de regeneración y un pseudodivertículo. La resonancia magnética cervical reportó una zona focal de engrosamiento e hiperintensidad T2 en la mucosa cervical esofágica. A la tercera semana se practicó una serie esofagogastroduodenal que confirmó la existencia de un pseudodivertículo esofágico a la altura de C2-C3, sin trayecto fistuloso; se le indicó dieta líquida con progresión a dieta normal, que toleró de manera adecuada. Permaneció en vigilancia durante 24 horas más y se dio de alta de la institución con estridor leve, 24 días posteriores a su ingreso.

A las seis semanas posteriores al primer estudio endoscópico, se realizó uno de control en el que se identificó una cicatriz lineal eritematosa, de $3 \mathrm{~mm}$ de largo, con tejido de regeneración (Figura 3B). Posteriormente evolucionó con buena tolerancia de la vía oral y ausencia de síntomas respiratorios.

\section{DISCUSIÓN}

La ingestión accidental de cuerpos extraños, sobre todo de batería de botón, representa un reto diagnóstico en los casos donde el evento no es presenciado por los cuidadores; incluso, en los que existe sospecha fundada y en pacientes menores de 3 años. Los síntomas suelen ser evidentes en el momento de la ingestión y, posteriormente, 
inespecíficos o asintomáticos, dependiendo del cuerpo extraño. Los síntomas respiratorios, sobre todo estridor $y$ tos persistente sin datos de proceso infeccioso, con manifestaciones gastrointestinales: vómitos o rechazo a la alimentación que obligan a establecer el diagnóstico diferencial con estudios de extensión: radiografías de tórax y abdomen anteroposterior y lateral, sobre todo cuando el estridor laríngeo no disminuye en las primeras 24 a 36 horas de evolución con tratamiento médico "adecuado". 4,5,7

La mayor parte de las ingestiones de cuerpos extraños, incluidas las baterías de botón, suceden en lactantes y preescolares ${ }^{6-10}$ quienes en su afán constante de exploración ingieren cuerpos extraños, de los que solo 3 a 10\% tendrán manifiestaciones clínicas, dependientes del tamaño y localización de los cuerpos. ${ }^{11,12}$ El sitio más frecuente de impactación es el tercio superior del esófago, en el borde inferior del músculo cricofaríngeo, que representa 60 a $75 \%$ de los casos. ${ }^{13,14,15}$ En el servicio de Gastroenterología y Nutrición pediátrica se reporta una frecuencia de cuerpos extraños en el esófago superior a 23.7\%, esófago medio $9.2 \%$, esófago inferior 3.9\%, y supraesofágico $6.6 \%$, de 76 pacientes a quienes se les ha practicado extracción de cuerpo extraño mediante panendoscopia (2013 a 2018). ${ }^{16}$

Por lo que se refiere a las manifestaciones clínicas, éstas incluyen: vómito (33.3\%), odinofagia $(22.9 \%)$, sialorrea $(22.9 \%)$, tos $(18.8 \%)$, dificultad respiratoria $(12.5 \%)$, atragantamiento $(10.4 \%)$, disfagia $(10.4 \%)$, anorexia $(8.3 \%)$, asfixia $(2 \%)$, hematemesis $(2 \%)^{17}$ y rechazo a la alimentación. El estridor se ha descrito en reportes de casos como consecuencia de paresia o parálisis de las cuerdas vocales, sin establecerse una prevalencia exacta. ${ }^{4,5,7,18}$ En el caso de la batería de botón es posible observar la imagen de doble contorno en la proyección anteroposterior y bordes redondeados y de aspecto "escalonado" (step off) en la proyección lateral.7,8,9

Se han descrito diferentes mecanismos implicados en la lesión de la mucosa esofágica: a) cáustica, por liberación de electrolitos alcalinos

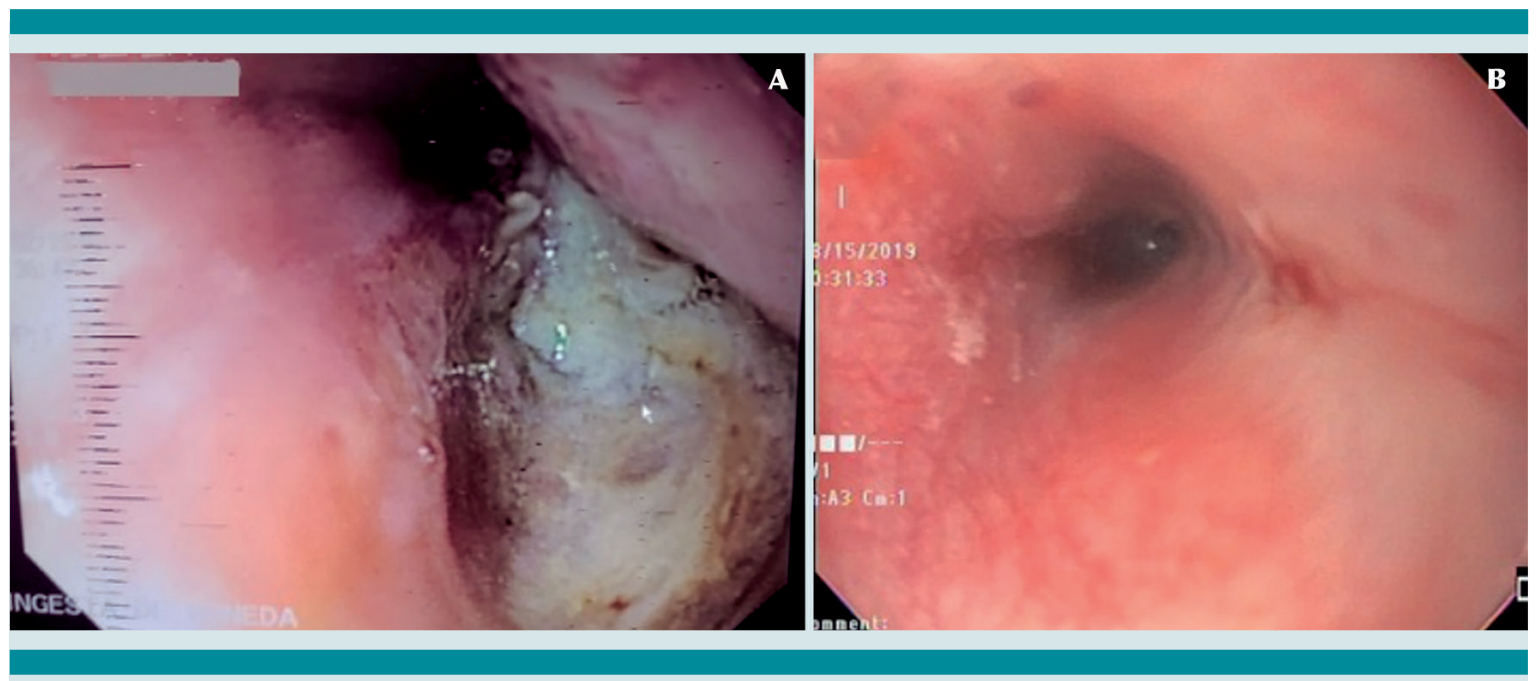

Figura 3. A) Úlcera con membranas blanquecinas y zonas de necrosis a $10 \mathrm{~cm}$ de la arcada dental superior donde previamente se encontraba alojado el cuerpo extraño (batería de botón), clasificándose como esofagitis por caústicos grado IIB de Zargar. B) Cicatriz lineal en la zona donde previamente se encontraba la úlcera en el control endoscópico realizado 6 semanas posteriores a la hospitalización. 
Baños-Rocha LM, et al. Ingestión de cuerpo extraño atípica en el lactante

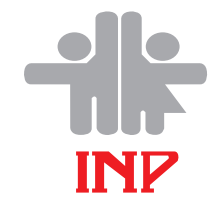

(hidróxido de sodio y potasio) cuando la batería entra en contacto con el ambiente húmedo del cuerpo humano; b) eléctrica, por quemadura debido a la corriente inducida entre la mucosa y una batería de botón con carga residual; c) térmica o tóxica por absorción local de litio o mercurio de la batería de botón; d) mecánica, por necrosis por presión directa en el tejido adyacente y por producción de álcali "de novo" desde la corriente externa. ${ }^{6,8,10,12,14-19}$

Las guías internacionales recomiendan la extracción inmediata del cuerpo extraño, sobre todo si se localiza en el esófago, porque existe un riesgo considerable de lesión en menos de 2 horas luego de su ingesta. $6,7,9,11,17,18$ La severidad de la lesión puede ir desde erosiones superficiales en la mucosa (menores) hasta amplios segmentos de necrosis (mayores). Las complicaciones mayores se han reportado entre 0.35 a $4 \%$, con mortalidad de $0.15 \%::^{12,17}$ fístulas traqueoesofágicas $(47.9 \%)$, perforación esofágica (23.3\%), estenosis esofágica $(38.4 \%)$, parálisis de la cuerda vocal $(9.6 \%)^{9}$ y entre otras menos frecuentes: mediastinitis, espondilodiscitis, estenosis traqueal, neumonía aspirativa y fístula aortoentérica; esta última puede dar lugar a hemorragia masiva y conducir a la muerte. ${ }^{5,7,11,12}$

Los factores de riesgo de complicaciones dependen de:

- Composición y tamaño: litio y mayores de $20 \mathrm{~mm}$

- Voltaje: de $3.0 \mathrm{~V}$.

- Edad menor de 4 años

- Cantidad: más de una batería de botón

- Tiempo de ingestión: mayor a 2 horas o desconocido

- Diagnóstico y tratamiento: inapropiado o retraso en la extracción de la batería de botón. 5,10,19-21
La ingestión de una batería de botón es una emergencia (extracción menor a 2 horas), si tiene síntomas y localización esofágica se procede, de inmediato, a la endoscopia para retirarla, ${ }^{22}$ y a la evaluación y clasificación de las lesiones de la mucosa posterior al retiro para normar la conducta terapéutica. ${ }^{23,24,25}$ La importancia de la detección de estos eventos radica en la identificación y tratamiento oportunos con el propósito de disminuir la morbilidad y mortalidad asociadas.

Si bien en las guías internacionales no se cuenta con un procedimiento unificado para la conducta médica posterior a la extracción endoscópica de las baterías de botón, , ,8,9,10,12 en el servicio de Gastroenterología y Nutrición los procedimientos se determinan según la gravedad de las lesiones. En el caso de las baterías de botón, el mecanismo más significativo de lesión tisular es la acumulación de iones de hidróxido que lleva a la licuefacción de los tejidos y necrosis, comparable con una lesión por cáusticos alca$\operatorname{linos}^{26}$ y se determina la estratificación según la clasificación de Zargar. ${ }^{27-31}$ Cuadro 1

Los esteroides se indican para prevención de estenosis en pacientes con esofagitis por cáusticos debido a su mecanismo de reducción de la proliferación de fibroblastos. Su prescripción es motivo de controversia porque los metanálisis no habían demostrado beneficio; $;^{32,33,34} \sin$ embargo, en 2014 se publicó un estudio experimental prospectivo ${ }^{35}$ en el que se redujo de forma significativa la necesidad de dilatación endoscópica en los pacientes con esofagitis grado IIB, lo que justifica la indicación de la metilprednisolona en la práctica clínica. Aunque no haya recomendaciones precisas en este contexto, en el paciente del caso, la metilprednisolona facilitó la evolución endoscópica y clínica. 
Cuadro 1. Clasificación de Zargar de ingestión de cáusticos

\begin{tabular}{|c|c|c|c|}
\hline Grado & Características endoscópicas & Pronóstico & Conducta médica \\
\hline 0 & Mucosa normal o sin lesiones & Bueno, sin secuelas & $\begin{array}{l}\text { Inicio inmediato de la vía oral si tolera egreso } \\
\text { a domicilio }\end{array}$ \\
\hline I & Edema e hiperemia de la mucosa & Bueno, sin secuelas & $\begin{array}{l}\text { Inicio inmediato de la vía oral si tolera egreso } \\
\text { a domicilio }\end{array}$ \\
\hline II A & $\begin{array}{l}\text { Exudados, erosiones, úlceras superficia- } \\
\text { les y hemorragia, lesiones no circunfe- } \\
\text { renciales o pseudomembranosas }\end{array}$ & $\begin{array}{l}\text { Bueno. Riesgo de estenosis } \\
\text { menor de } 5 \%\end{array}$ & $\begin{array}{l}\text { Inicio inmediato de la vía oral, si tolera egreso } \\
\text { a domicilio. Considerar toma de serie EGD a } \\
\text { la tercera semana. }\end{array}$ \\
\hline IIB & $\begin{array}{l}\text { Úlceras circunferenciales o profundas } \\
\text { o necrosis }\end{array}$ & $\begin{array}{l}\text { Riesgo de estenosis } \\
\text { mayor de } 71.4 \%\end{array}$ & $\begin{array}{l}\text { Admisión. Esteroides, antibióticos, ayuno, } \\
\text { sonda para alimentación vs NPT serie EGD } \\
\text { tercera semana. }\end{array}$ \\
\hline IIIA & $\begin{array}{l}\text { Pequeñas áreas aisladas de necrosis, } \\
\text { lesiones circunferenciales o pseudo- } \\
\text { membranosas }\end{array}$ & $\begin{array}{l}\text { Riesgo de estenosis de } \\
\text { 95\%. Complicaciones } \\
\text { agudas } 19 \%\end{array}$ & $\begin{array}{l}\text { Admisión a terapia intensiva, ayuno, líquidos } \\
\text { endovenosos, antibióticos de amplio espec- } \\
\text { tro, IBP, sonda para alimentación vs NPT. } \\
\text { Serie EGD tercera semana. }\end{array}$ \\
\hline IIIB & Extensas áreas de ulceración o necrosis & $\begin{array}{l}\text { Mortalidad } 64.7 \% \text {. } \\
\text { Riesgo de estenosis } 100 \%\end{array}$ & $\begin{array}{l}\text { Cirugía de urgencia, admisión a terapia inten- } \\
\text { siva, ayuno, líquidos endovenosos, antibióticos } \\
\text { de amplio espectro, IBP. }\end{array}$ \\
\hline
\end{tabular}

Adaptado de Zargar, Methasate, Baskin, Temiz, De Lusong. ${ }^{27-31}$ NPT: Nutrición parenteral. IBP: Inhibidor de bomba de protones. EGD: Esofagogastroduodenal.

En lo que concierne a los antibióticos y ayuno, está descrita su indicación en las guías de tratamiento de pacientes que ingieren baterías de botón, en quienes se documente lesión de la mucosa significativa o en proximidad con la aorta, con toma de imágenes complementarias para excluir fístulas, previo a la progresión de la dieta. 9,36,37

\section{CONCLUSIÓN}

El estridor agudo y la tos acompañados de manifestaciones gastrointestinales: vómito y rechazo a la alimentación en lactantes y preescolares sin antecedente de infección, obligan a descartar la existencia de cuerpos extraños y a practicar estudios de extensión y extracción endoscópica oportuna, sobre todo en el caso de las baterías de botón.

\section{REFERENCIAS}

1. Escobar NL, Needleman J. Stridor. Pediatr Rev. 2015; 39 (6). doi. 10.1542/pir.2017-0157
2. Marquez YA, et al. Estridor persistente. Neumol Pediatr. 2018; 13 (1): 24-28. http://www.neumologia-pediatrica. cl/wp-content/uploads/2018/02/estridor_persistente.pdf.

3. Mandal A, et al. Upper airway obstruction in children. Indian J Pediatr. 2015; 82 (8): 737-44. doi:10.1007/s12098015-1811-6

4. Celmina M, Paule S. Stridor in children. Breathe (Sheff). 2018; 14 (3): e111-e117. doi:10.1183/20734735.017018

5. Wallace $B$, et al. Button battery ingestion complications. J Pediatr Surg Case Reports. 2017; 19: 1-3. doi.10.1016/j. epsc.2016.12.009

6. Litovitz T, et al. Preventing battery ingestions: An analysis of 8648 cases. Pediatrics. 2010; 125 (6): 1178-83. doi.10.1542/peds.2009-3038

7. Leinwand $\mathrm{K}$, et al. Button battery ingestion in children: $\mathrm{A}$ paradigm for management of severe pediatric foreign body ingestions. Gastrointest Endosc Clin N Am. 2016; 26 (1): 99-118. doi.10.1016/j.giec.2015.08.003.

8. Lahmar J, et al. Esophageal lesions following button-battery ingestion in children: Analysis of causes and proposals for preventive measures. Eur Ann Otorhinolaryngol Head Neck Dis. 2018; 135 (2): 91-94. doi. 10.1016/j.anorl.2017.09.004.

9. Kramer RE, et.al. North American Society for Pediatric Gastroenterology, Hepatology, and Nutrition Endoscopy Committee. Management of ingested foreign bodies in children: a clinical report of the NASPGHAN Endoscopy Committee. J Pediatr Gastroenterol Nutr. 2015; 60 (4): 562-74. doi. 10.1097/MPG.0000000000000729. 
Baños-Rocha LM, et al. Ingestión de cuerpo extraño atípica en el lactante

10. Cairns R, et.al. Button battery exposures in Australian children: a prospective observational study highlighting the role of poisons information centres. Clin Toxicol (Phila). 2019; 57 (6): 404-10. doi. 10.1080/15563650.2018.1537492.

11. Abdulkareem I, et al. Button battery induced traumatic tracheoesophageal fistula: Case report and review of literature. Sudan J Paediatr. 2011; 11 (2): 43-49.

12. Amanatidou $\mathrm{V}$, et al. Button battery ingestion. Pediatr Emer Care. 2011; 27: 186-88

13. Littlehales $E$, et al. Double button battery ingestion. The "macaroon" sign. J Pediatr Surg Case Reports. 2018; 36: 36-39. doi:10.1016/j.epsc.2018.06.013.

14. Kalyanshettar SS, et al. Button battery ingestion-case report and review. J Clin Diagnostic Res. 2014; 8 (9): PD01-PD02. doi:10.7860/JCDR/2014/9707.4847

15. Green SS. Ingested and aspirated foreign bodies. Pediatrics in Review. 2015; 36 (10): 430-37.

16. Navia López LA, Cadena León JF. Características clínicas, radiológicas, endoscópicas de pacientes con diagnóstico de ingestión de cuerpo extraño en el Servicio de Gastroenterología en el Instituto Nacional de Pediatría entre enero 2013 a diciembre de 2018. Tesis para obtener el grado de especialista en Medicina Gastroenterología y Nutrición Pediátrica, Instituto Nacional de Pediatría, Universidad Autónoma de México, Ciudad de México.

17. Medina-Gaviria V, et al. Experiencia en el manejo de ingesta de pilas en un hospital pediátrico. Cir Pediatr. 2018; 31 (3): 121-24.

18. Patel SA, et al. Battery ingestion leading to bilateral vocal cord paresis. JAMA Otolaryngol Head Neck Surg. 2013; 139 (3): 304-6. doi.10.1001/jamaoto.2013.1825

19. Völker J, et.al. Pathophysiology of esophageal impairment due to button battery ingestion. Int J Pediatr Otorhinolaryngol. 2017; 100: 77-85. doi. 10.1016/j.ijporl.2017.06.030.

20. Macchini $F$, et al. Button battery ingestion in children: $A$ specific clinical issue. Clin Endosc. 2018; 51 (6): 602-3. doi.10.5946/ce.2018.137

21. Thatcher A, et al. Complication of button battery ingestion. Gastroenterology. 2017; 153 (5): e10-e11. doi. 10.1053/j. gastro.2017.03.061

22. Cadena-León JF, et al. Manejo de la ingesta de cuerpos extraños por vía digestiva. Acta Pediatr Mex. 2109; 40 (5): 290-94.

23. Geng $C$, et al. Endoscopic management of foreign bodies in the upper gastrointestinal tract: a retrospective study of 1294 cases. Scand J Gastroenterol. 2017; 52 (11): 1286-91. doi.10.1080/00365521.2017.1350284
24. National poison data system Button Battery Ingestion Statistics [Internet]. Washington DC, USA. http://www. poison.org/battery/stats

25. Betalli P, et al. Update on management of caustic and foreign body ingestion in children. Diagn Ther Endosc. 2009; 2009. doi. 10.1155/2009/969868

26. Jatana KR, et al. Pediatric button battery injuries: 2013 task force update. Int J Pediatr Otorhinolaryngol. 2013; 77 (9): 1392-99. doi.10.1016/j.ijporl.2013.06.006

27. Ali Zargar S, et al. The role of fiberoptic endoscopy in the management of corrosive ingestion and modified endoscopic classification of burns. Gastrointest Endosc. 1991; 37 (2): 165-69. doi.10.1016/S0016-5107(91)70678-0

28. Role of endoscopy in caustic injury of the esophagus. World J Gastrointest Endosc. 2018; 10 (10): 274-82. doi. 10.4253/ wjge.v10.i10.274

29. Baskin D, et.al. A standardized protocol for the acute management of corrosive ingestion in children. Pediatr Surg Int. 2004; 20 (11-12): 824-8. doi. 10.1007/s00383-004-1294-4.

30. Temiz A, et al. Predictability of outcome of caustic ingestion by esophagogastroduodenoscopy in children. World J Gastroenterol. 2012; 18 (10): 1098-103. doi.10.3748/ wjg.v18.i10.109

31. De Lusong $M$, et al. Management of esophageal caustic injury. World J Gastrointest Pharmacol Ther 2017; 8 (2): 90-98. doi.10.4292/wjgpt.v8.i2.90

32. Pelclová D, Navrátil T. Do corticosteroids prevent oesophageal stricture after corrosive ingestion? Toxicol Rev. 2005; 24 (2): 125-29. doi.10.2165/00139709-200524020-00006

33. Fulton JA, Hoffman RS. Steroids in second degree caustic burns of the esophagus: A systematic pooled analysis of fifty years of human data: 1956-2006. Clin Toxicol. 2007; 45 (4): 402-8. doi.10.1080/15563650701285420

34. Bird JH, et al. Controversies in the management of caustic ingestion injury: an evidence-based review. Clin Otolaryngol. 2017; 42 (3): 701-8. doi.10.1111/coa.12819

35. Usta $\mathrm{M}$, et al. High doses of methylprednisolone in the management of caustic esophageal burns. Pediatrics. 2014; 133 (6): e1518-24. doi.10.1542/peds.2013-3331

36. Kurowski JA, Kay M. Caustic ingestions and foreign bodies ingestions in pediatric patients. Pediatr Clin North Am. 2017; 64 (3): 507-24. doi.10.1016/j.pcl.2017.01.004

37. Bolton SM, et al. Button battery and magnet ingestions in the pediatric patient. Curr Opin Pediatr. 2018; 30 (5): 653-59. doi.10.1097/MOP.0000000000000665 